\title{
Inhibition of the Amyloidogenesis of Transthyretin by Natural Products and Synthetic Compounds
}

\author{
Takeshi Yokoyama $^{a}$ and Mineyuki Mizuguchi*,a,b \\ ${ }^{a}$ Graduate School of Medicine and Pharmaceutical Sciences, University of Toyama; 2630 Sugitani, \\ Toyama 930-0194, Japan: and ${ }^{b}$ Graduate School of Innovative Life Science, University of Toyama; \\ 2630 Sugitani, Toyama 930-0194, Japan. \\ Received February 27, 2018
}

\begin{abstract}
Hereditary transthyretin (TTR)-related amyloidosis is caused by mutations in the TTR gene. The mutations destabilize the tetramer and/or monomer of TTR, and thus the stabilization of TTR is a key strategy for the treatment of TTR-related amyloidosis. In this review, we summarized the natural products and synthetic compounds that have been shown to inhibit the amyloidogenesis of TTR. The stabilizers and/or the amyloid fibril disrupters isolated from natural sources may become lead compounds for the treatment of TTR-related amyloidosis.
\end{abstract}

Key words transthyretin; crystal structure; amyloidosis; natural product; stabilizer; amyloid fibril disrupter

\section{INTRODUCTION}

Transthyretin (TTR) is a $\beta$-sheet-rich homo-tetrameric protein whose subunit is composed of 127 amino acid residues. The vast majority of TTR is produced by the liver and is secreted into the blood. ${ }^{1)}$ TTR is also expressed in the choroid plexus of the brain, the retinal pigment epithelium of the eye, and the $\alpha$-cells of pancreatic islets. ${ }^{2)}$ TTR transports retinol via binding to holo-retinol binding protein in the blood. TTR also binds and transports thyroxine (T4) in the blood and cerebrospinal fluid. ${ }^{1)}$ The TTR tetramer has two thyroxine-binding channels in the dimer-dimer interface (Fig. 1a). However, more than $99 \%$ of the T4-binding sites in TTR are unoccupied in the blood, since T4 predominantly binds to thyroid binding globulin and albumin. The majority of the T4-binding sites in TTR are also unoccupied in the cerebrospinal fluid because of the low concentration of T4, although TTR is the main carrier of T4 in the cerebrospinal fluid. ${ }^{1)}$

\section{TTR-RELATED AMYLOIDOSIS}

TTR causes transthyretin-related amyloidosis (ATTR), including familial amyloid polyneuropathy (FAP), familial amyloid cardiomyopathy (FAC), central nervous system selective amyloidosis (CNSA), and senile systemic amyloidosis (SSA). ${ }^{2,3)}$ Hereditary ATTR (FAP, FAC, and CNSA) is an autosomal dominant disease that is caused by mutations in the TTR gene. Almost all of the patients are heterozygotes, namely, their TTR tetramers are composed of mutant and/or wild-type subunits. ${ }^{1}$ To date, more than 130 mutations have been identified in the TTR gene, and most of them are associated with the onset of ATTR. ${ }^{2}$ Hereditary ATTR is a progressive and devastating disease. It can be seen in people as young as their late twenties, and the average life expectancy after the onset of symptoms is about 10 years. ${ }^{2,4)}$ The clinical features of ATTR include sensorimotor polyneuropathy, autonomic dysfunction, heart and kidney failure, gastrointestinal tract disorders, vitreous opacity, cerebral infarction, and dementia. ${ }^{2,5,6)}$ Amyloid fibrils of TTR are deposited in the nerves, heart, kidney, gastrointestinal tract, retina, leptomeninges, etc. ${ }^{2,6)}$

The TTR tetramer is highly stable under physiological conditions, but its dissociation to monomers and subsequent partial unfolding results in aggregation of the amyloidogenic intermediate, leading to the accumulation of amyloid fibrils and oligomers that ultimately cause the clinical symptoms of ATTR. ${ }^{1)}$ The mutations associated with ATTR induce subtle structural changes that destabilize the TTR tetramer and/or monomer, thereby promoting dissociation into monomers and/or subsequent partial unfolding. The most frequent mutant associated with ATTR is V30M-TTR, which is found worldwide as well as in endemic areas in Portugal, Japan, and Sweden. ${ }^{5)}$ The V30M mutation makes TTR less stable and more susceptible to tetramer dissociation into monomers. ${ }^{7)}$ Therefore, stabilization of the tetramer is a key strategy for the treatment of ATTR.

Stabilization of the TTR tetramer and the inhibition of TTR amyloidogenesis can also be achieved by small molecules that bind to the T4-binding sites of TTR ${ }^{8-10)}$ (Figs. 1a, e). A number of structurally diverse small molecules that stabilize the TTR tetramer, and thereafter inhibit the resulting amyloidogenesis, have been reported (Fig. 2). This review describes the biophysical properties of natural products and synthetic compounds that inhibit the amyloidogenesis of TTR.

\section{NATURAL PRODUCT INHIBITORS OF ATTR}

Investigation of the interactions of TTR with natural products was already underway when, in 1978, it was demonstrated that TTR was associated with ATTR. ${ }^{11)}$ At that time, TTR was known as T4-binding prealbumin (TBPA), and the research was conducted from the point of view of the interaction between the constituents of blood and various drug ingredients. In 1963, salicylic acid was indicated to competitively bind to TTR with T4 by a competitive binding assay using isotopic labeled T4. ${ }^{12}$ In 1986, phloretin, which is a natural polyphenol chalcone found in apple tree leaves, was also shown to bind to TTR in a competitive manner with T4. ${ }^{13)}$ In 1994, retinoic 
(a)
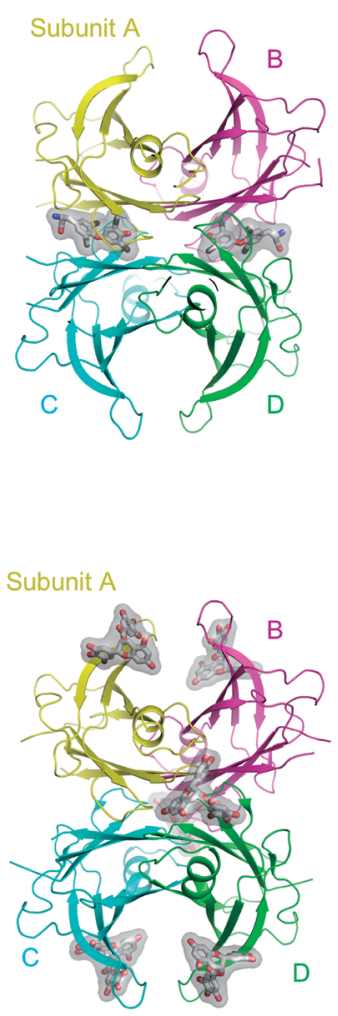

(c)

$\varphi^{90^{\circ}}$
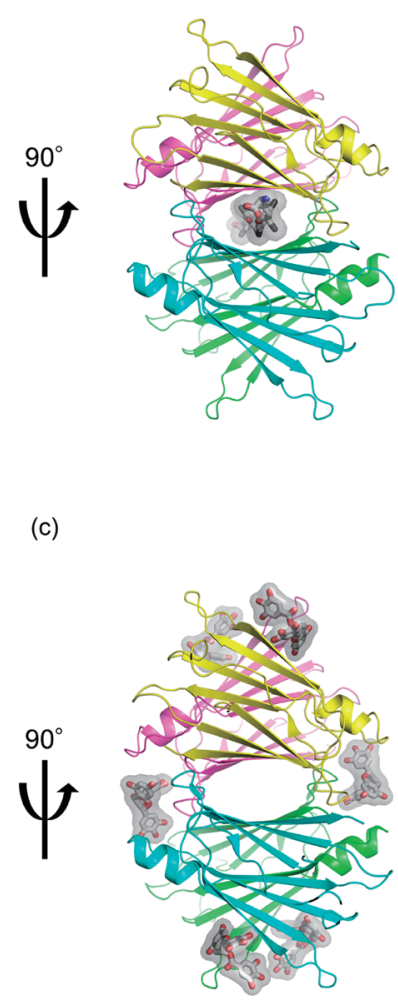

(b)

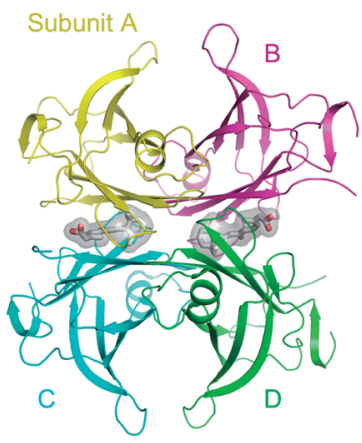

(d)

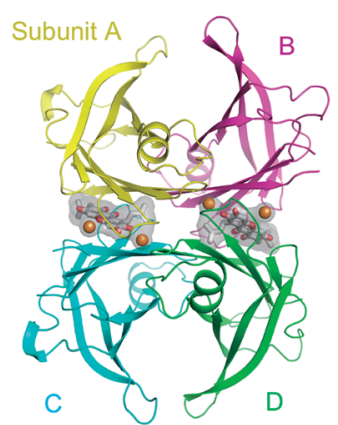

(e)

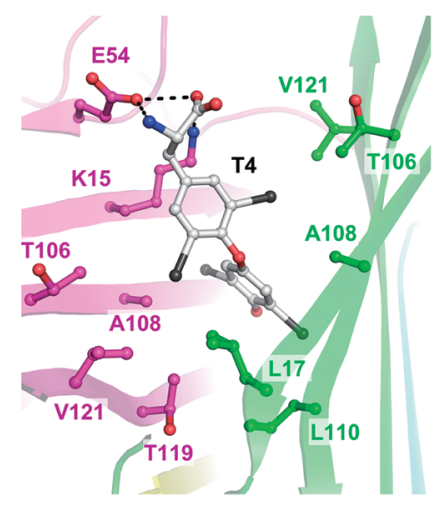

(f)

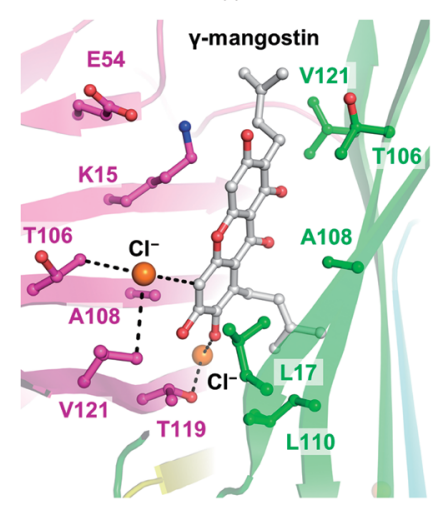

Fig. 1. (a-d) X-Ray Crystal Structures of TTR in Complex with (a) T4 (PDB ID: 2ROX), (b) Retinoic Acid (PDB ID: 1TYR), (c) EGCG (PDB ID: $3 \mathrm{NG5}$ ) and (d) $\gamma$-Mangostin (PDB ID: 4Y9E)

Four subunits of TTR are designated as A-D and colored yellow, magenta, cyan and green, respectively. The stabilizers are represented as a stick model with a semitransparent surface. Chloride ions are represented as orange spheres. (e, f) Schematic view of the interactions of TTR with (e) T4 and (f) $\gamma$-mangostin. The carbon atoms of subunits B and D and stabilizers are colored magenta, green and white, respectively. Iodine atoms of T4 are colored grey. Black dashed lines indicate hydrogen bonds, $\mathrm{CH}{ }^{\cdots} \mathrm{Cl}^{-}$bonds or $\mathrm{OH}^{\cdots} \mathrm{Cl}^{-}$bonds.

\section{Biography}

Takeshi Yokoyama graduated from the School of Engineering Science, Osaka University (2001), and the Graduate School of Integrated Science, Yokohama City University (2006). He conducted postdoctoral research at the Department of Biology and Biochemistry, University of Houston, TX, U.S.A. (2006-2008), and at the Frontier Research Center for Applied Atomic Sciences, Ibaraki University (2008-2011). In 2011, he became assistant professor at the Laboratory of Structural Biology, Graduate School of Medicine and Pharmaceutical Sciences, University of Toyama. Currently, he is working on neutron protein crystallography of the protein-inhibitor complex, focusing on the role of hydrogens, and on drug discovery for disease-related proteins such as transthyretin, kinase and bromodomain.

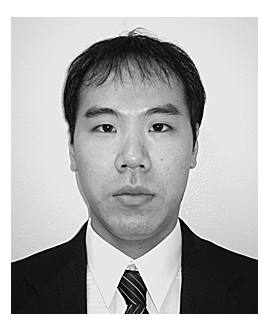

Takeshi Yokoyama 


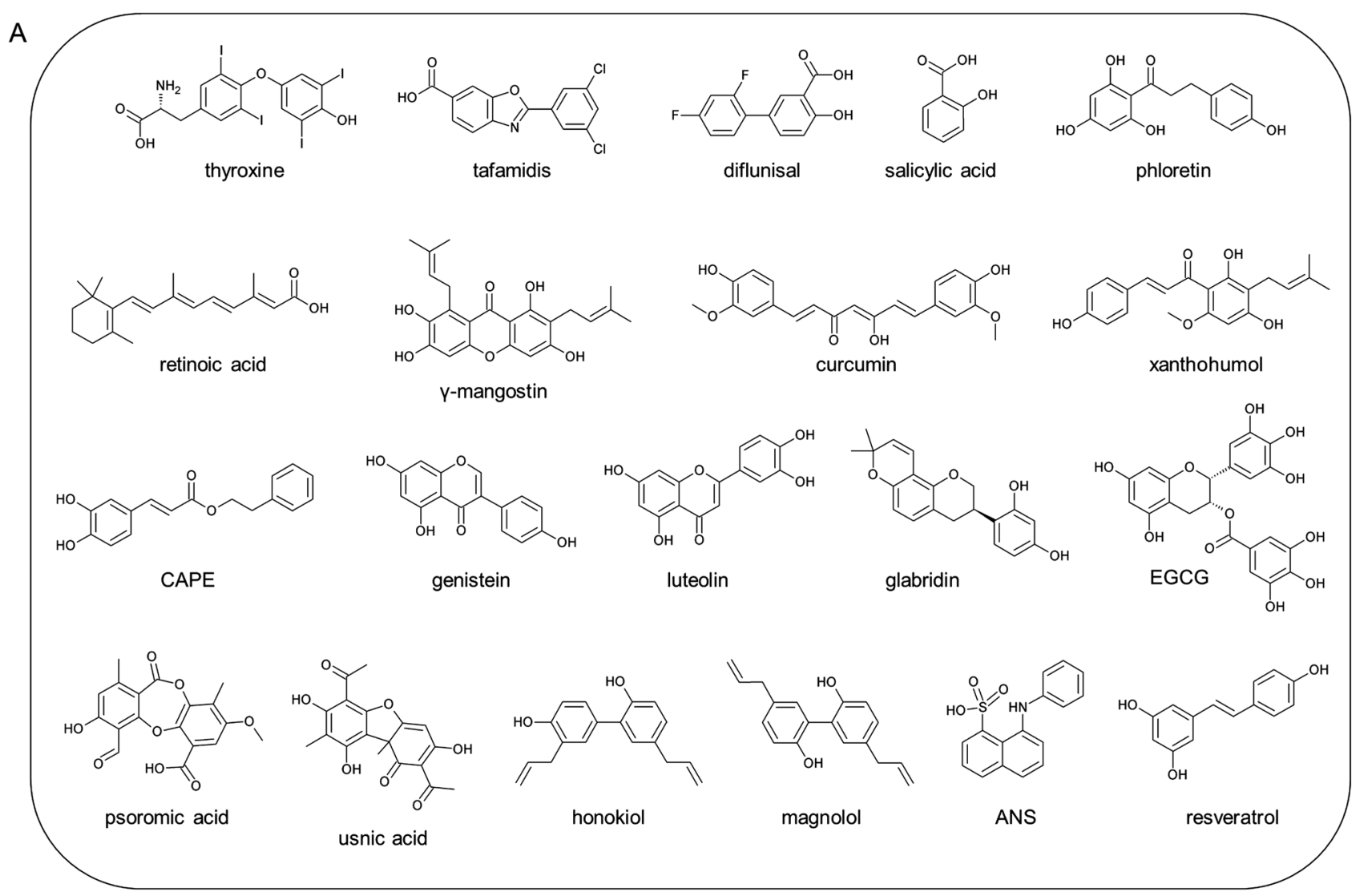

B

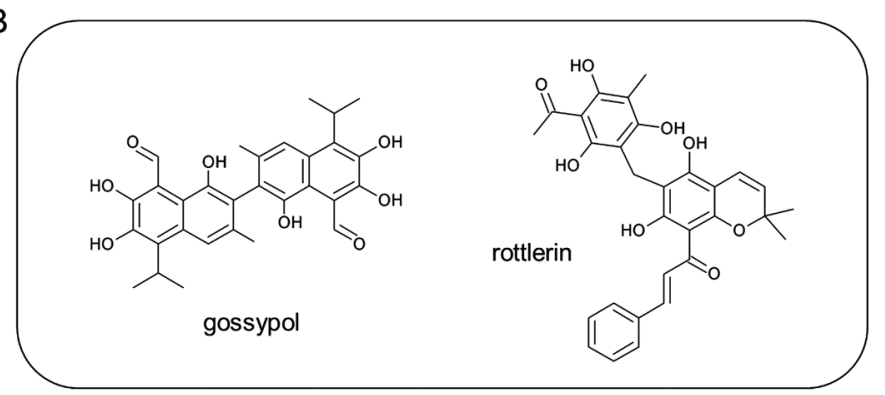

C

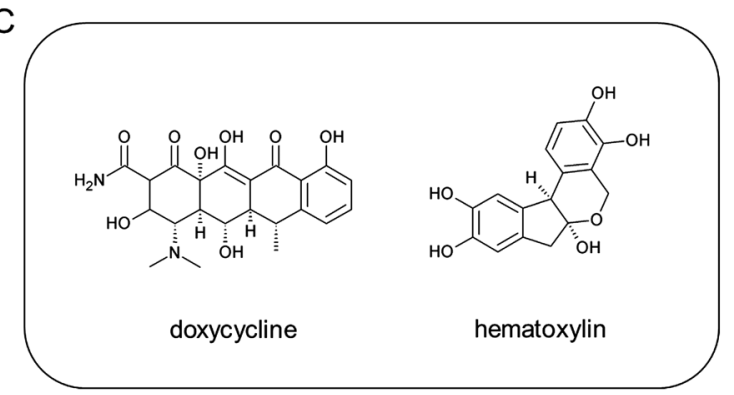

Fig. 2. Chemical Structures of the TTR Amyloidogenesis Inhibitors

The compounds in group A have been shown to stabilize the TTR tetramer (TTR stabilizer). The compounds in group B were shown to exhibit both TTR stabilization and TTR amyloid fibril disruption activities in the present study (dual inhibitors). The compounds in group C have been shown to disrupt the TTR amyloid fibril (fibril disrupters).

acid, which is a metabolite of retinol, was shown to bind to $\mathrm{TTR}$ in a competitive manner with $\mathrm{T} 4,{ }^{14)}$ and in the following year, the X-ray crystal structure of TTR in a complex with retinoic acid was solved at $1.8 \AA$ resolution $^{15)}$ (Fig. 1b). This crystallographic analysis provided the first picture of TTR in a complex with a natural product other than T4. In 1996, it was elucidated that the binding of T4 stabilizes the TTR tetramer and inhibits the amyloid fibril formation of TTR. ${ }^{8)}$ By this discovery, the search for TTR stabilizers that bind to the T4binding site of TTR and stabilize the TTR tetramer became an efficient strategy for TTR amyloidosis therapy. ${ }^{16)}$ In 1998, 77 compounds containing natural flavonoids and anthraquinones were screened for their inhibitory activity against TTR amyloid fibril formation. ${ }^{9)}$ At that time, it was understood that the common structural feature of TTR stabilizers, which are able to bind to the T4-binding site of TTR, was a two aromatic-ring substructure with a linker. In 2005, the xanthonoids from $\mathrm{Ca}$ lophyllumteysmannii var. inophylloide were shown to bind to
TTR in a competitive manner with T4, and the binding modes were predicted by docking simulation. ${ }^{17)}$ In terms of xanthone derivatives, we identified $\gamma$-mangostin, which is found in the pericarp of mangosteen, as a TTR amyloid fibril formation inhibitor. ${ }^{18)}$ The crystallographic analysis of TTR and the $\gamma$-mangostin complex revealed a novel binding mode; that is, the binding of $\gamma$-mangostin was associated with the binding of a chloride ion, suggesting that $\gamma$-mangostin is a novel TTR amyloidogenesis inhibitor which cooperatively inhibits TTR amyloid fibril formation with the help of chloride ions (Figs. 1c, f). In 2014, we also found that caffeic acid phenethyl ester (CAPE) from New Zealand propolis inhibits TTR amyloid fibril formation. ${ }^{19)}$ Some compounds found in common and functional foods have also been identified as TTR amyloidogenesis inhibitors. Curcumin from turmeric and xanthohumol from hops were shown to inhibit TTR amyloid fibril formation in 2009 and 2010, respectively. ${ }^{20,21)}$ In addition, flavonoids should be introduced as a representative compound group that 
(a)

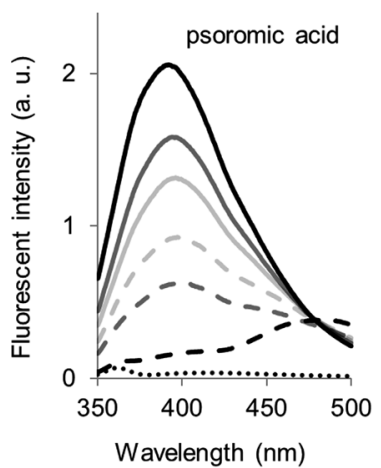

(e)

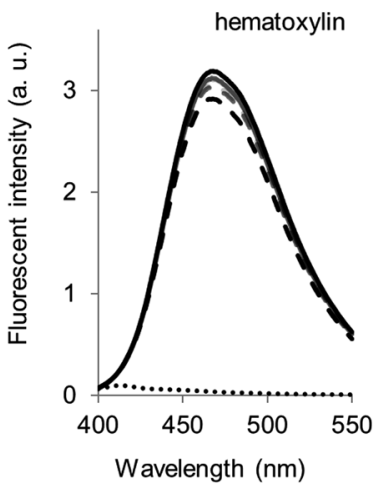

(b)

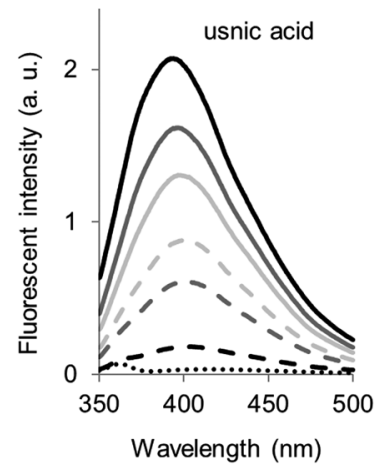

(f)

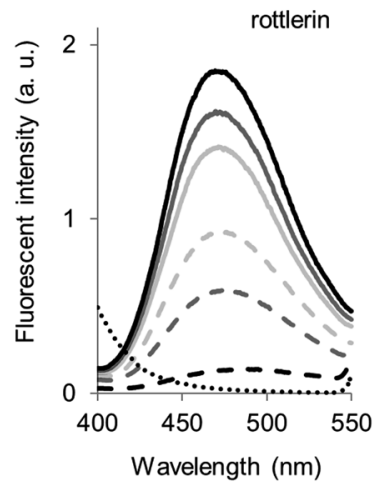

(c)

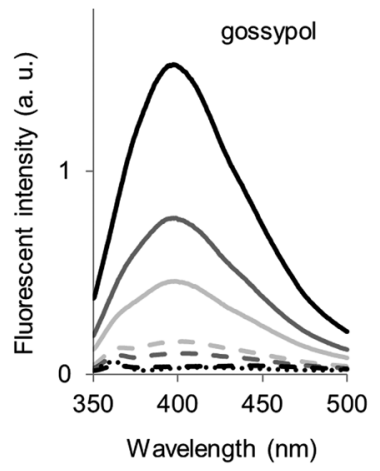

(g)

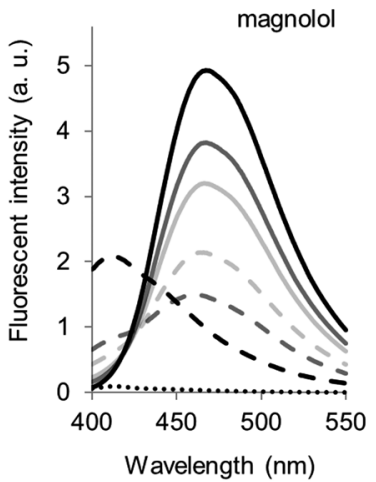

(d)

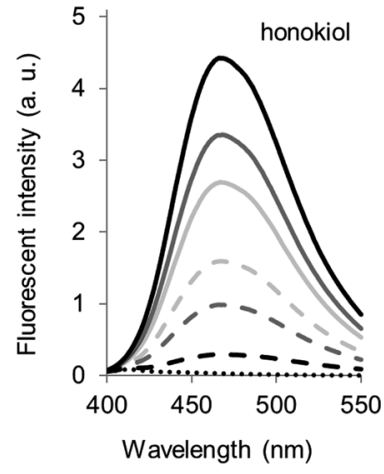

(h)

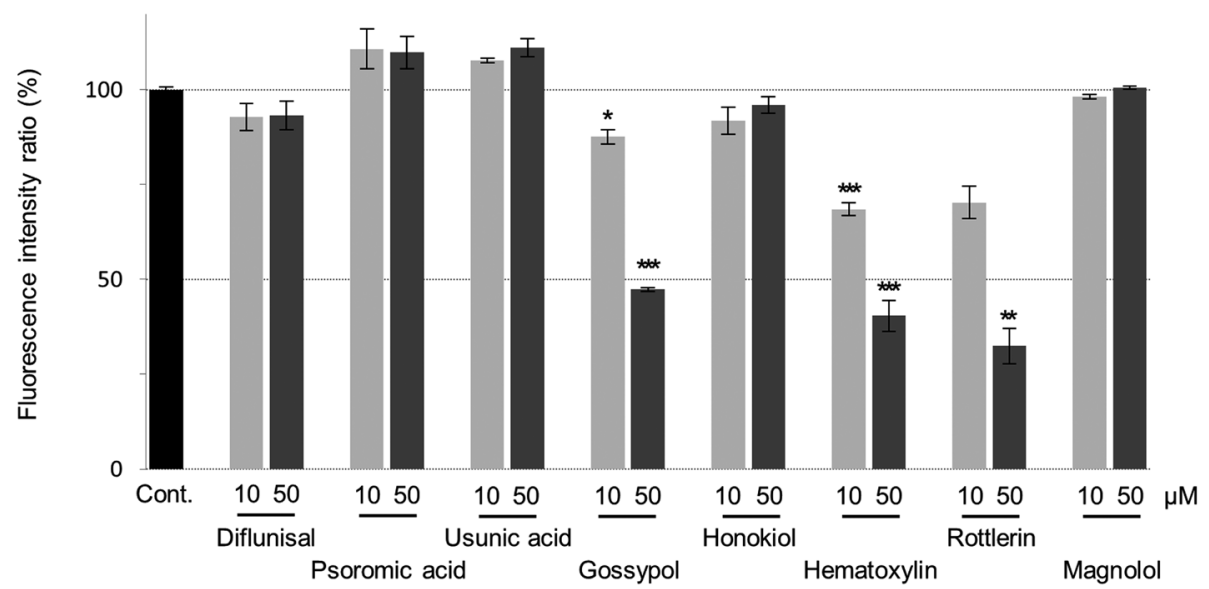

Fig. 3. (a-g) Competitive Binding Assays of the Selected Compounds with Fluorescent Probes

The titration and fluorescence experiments were performed as described previously. ${ }^{18)}$ Briefly, the compounds were titrated into a $2 \mu \mathrm{M}$ TTR solution in the order of the fluorescent probe and the natural products $(1-50 \mu \mathrm{M})$, and fluorescence spectra were recorded at each step. The measurement conditions (titrated compound, fluorescent probe and excitation wavelength) were (a) psoromic acid, $5 \mu \mathrm{m}$ resveratrol, $320 \mathrm{~nm}$, (b) usnic acid, $5 \mu \mathrm{m}$ resveratrol, $320 \mathrm{~nm}$, (c) gossypol, $5 \mu \mathrm{m}$ resveratrol, $320 \mathrm{~nm}$, (d) honokiol, $5 \mu \mathrm{m}$ ANS, $360 \mathrm{~nm}$, (e) hematoxylin, $5 \mu \mathrm{m}$ ANS, $360 \mathrm{~nm}$, (f) rottlerin, $40 \mu \mathrm{m}$ ANS, $280 \mathrm{~nm}$, and (g) magnolol, $5 \mu \mathrm{m}$ ANS, $360 \mathrm{~nm}$. The decrease in fluorescence intensity indicates that the selected compounds competitively displaced the probes from the T4-binding sites. (h) TTR amyloid fibril disaggregation assays. Amyloid fibril disaggregation assays were performed as described previously. ${ }^{19)}$ Briefly, preformed TTR amyloid fibrils were incubated in the presence of 10 or $50 \mu \mathrm{M}$ of the compounds for 5-6h, and the extent of amyloid fibril formation was quantified using a thioflavin T assay (excitation: $440 \mathrm{~nm}$; emission: $484 \mathrm{~nm}$ ).

exhibits inhibitory activity for TTR amyloid fibril formation. In 2005, genistein, which is an isoflavone found in soybeans, was shown to inhibit TTR amyloid fibril formation. ${ }^{22)}$ In 2012, it was elucidated that the inhibitory potency of luteolin was stronger than that of genistein. ${ }^{23)}$ In 2014, we found that glabridin, which is a prenylated isoflavan from licorice, is an inhibitor of TTR amyloidogenesis, and the inhibitory potency of glabridin is similar to that of diflunisal. ${ }^{24)} \mathrm{X}$-ray crystallo- graphic analysis revealed that the interactions between TTR and glabridin consist mainly of hydrophobic interactions. The characteristic structural change occurred on A108, which is formed by $\mathrm{CH} \cdots \pi$ interaction with the A-ring of glabridin. It was suggested that the stable binding of glabridin would be achieved by the induced fit.

As described above, several natural products that bind to the T4-binding site have been discovered. Meanwhile, an al- 
losteric ligand has also been discovered. A 2009 study indicated that (-)-epigallocatechin-3-gallate (EGCG) from green tea binds to TTR in an uncompetitive manner with T4 and inhibits TTR amyloid fibril formation. ${ }^{25)}$ In the following year, the X-ray crystal structure of TTR in complex with EGCG was determined at $1.7 \AA$ resolution, and it was revealed that six EGCG molecules bind to the surface of the TTR molecule but not the T4-binding site ${ }^{26}$ (Fig. 1c). The authors suggested that the binding to the molecular surface induces non-toxic aggregation of TTR and consequently inhibits the amyloid fibril formation.

As one of the therapeutic strategies for TTR amyloidosis, amyloid fibril disrupters, which directly disaggregate amyloid fibrils, have been developed. It has been determined that doxycycline, a tetracycline antibiotic, dissolves TTR amyloid fibrils. ${ }^{27)}$ Although the mechanism by which the fibril disruption occurs is not fully understood, it is proposed that amyloid fibril disrupters bind uniformly on the fibers, which leads to disruption at multiple sites. ${ }^{28)}$ Several compounds have been shown to disaggregate various amyloid fibrils other than TTR amyloid fibrils. The typical structure of an amyloid fibril disrupter is a multi-cyclic system such as doxycycline. Considering that the T4-binding site accepts tricyclic compounds, such as $\gamma$-mangostin, it is conceivable that there is also a dual inhibitor which inhibits TTR amyloidogenesis by both stabilizing the TTR tetramer and disaggregating TTR amyloid fibrils. Indeed, some biphenyl ether compounds have been shown to exhibit TTR stabilization and fibril disruption activities. ${ }^{29)}$ Therefore, we performed a limited screening to discover the dual inhibitors from 26 natural products. The screening revealed that psoromic acid, usnic acid, gossypol, honokiol, hematoxylin, rottlerin and magnolol inhibited amyloid fibril formation (Table 1). These compounds were tested to determine whether they competitively bound to the T4-binding site; this was done using fluorescent probes such as 1,8-anilinonaphthalene-8-sulfonic acid (ANS) or trans-resveratrol. The competitive binding assays indicated that all but hematoxylin competitively bound to the T4-binding site with ANS or resveratrol (Figs. 3a-g).

In order to analyze their amyloid fibril disaggregation activity, pre-formed amyloid fibrils were incubated in the presence of the compounds, and the amounts of amyloid fibrils were quantified. Gossypol, hematoxylin and rottlerin disrupted the pre-formed amyloid fibrils (Fig. 3h). Based on the above observations, we concluded that gossypol and rottlerin are dual inhibitors, psoromic acid, usnic acid, honokiol, and magnolol are TTR stabilizers, and hematoxylin is an amyloid fibril disrupter. The disaggregation activity of rottlerin is not surprising, since it was shown that rottlerin dissolved the amyloid fibrils of lysozymes. It is possible that the T4-binding site of TTR may not accept a large molecule such as rottlerin. It is also possible that rottlerin binds to the allosteric site, and stabilizes the TTR tetramer in that manner, as with EGCG. The binding of rottlerin to the allosteric site may cause a decrease in the binding affinity at the T4-binding site.

\section{DRUGS FOR ATTR}

In addition to the natural products described above, Kelly and colleagues have discovered that several non-steroidal anti-inflammatory drugs have potential as inhibitors of TTR
Table 1. Limited Screening against Amyloidogenesis of V30M-TTR

\begin{tabular}{|c|c|c|c|}
\hline Compound name & Inhibition ratio $(\%)$ & Compd. Conc. $(\mu \mathrm{M})$ & $\mathrm{IC}_{50}(\mu \mathrm{M})$ \\
\hline Diflunisal & 97 & 40 & 6.3 \\
\hline Bilobalide & -1.6 & 80 & \\
\hline Boldin & -11 & 40 & \\
\hline Colchicine & 12 & 40 & \\
\hline Psoromic acid & 92 & 40 & 8.9 \\
\hline Piperine & 16 & 40 & \\
\hline Usnic acid & 77 & 20 & 7.7 \\
\hline Cryptotanshinone & 8.2 & 32 & \\
\hline Gossypol & 94 & 40 & 9.9 \\
\hline Honokiol & 95 & 40 & 8.5 \\
\hline Nalidixic acid & -1.6 & 40 & \\
\hline Oridonin & -5.3 & 80 & \\
\hline Parthenolide & -9.3 & 40 & \\
\hline Silibinin & 12 & 40 & \\
\hline Sinomenin & 3.6 & 40 & \\
\hline Tanshinone I & 14 & 40 & \\
\hline Tetrandrine & 1.9 & 40 & \\
\hline Hematoxylin & 78 & 40 & 25 \\
\hline Limonin & 9 & 32 & \\
\hline Rottolerin & 70 & 40 & 23 \\
\hline Magnolol & 81 & 40 & 19 \\
\hline Magnolignan C & 13 & 80 & \\
\hline Coumestrol & 11 & 20 & \\
\hline Brefeldin A & 15 & 20 & \\
\hline Coptisine & 12 & 20 & \\
\hline Emodin & 9.1 & 20 & \\
\hline Auraptene & -6.7 & 20 & \\
\hline
\end{tabular}

The TTR amyloid fibril formation assay was performed according to the previous study. ${ }^{24)}$ Briefly, V30M-TTR was incubated in the presence of the compounds at $\mathrm{pH}$ 4.5 for $4 \mathrm{~d}$, and then the amyloid fibrils were quantified using thioflavin- $\mathrm{T}$ by fluorometry (excitation: $440 \mathrm{~nm}$; emission: $484 \mathrm{~nm}$ ).

amyloidogenesis. ${ }^{30-33)}$ The non-steroidal anti-inflammatory drug diflunisal, which has already been approved as a prescription drug in more than 40 countries, binds to the T4binding sites of TTR, stabilizes the tetramer, and inhibits the amyloidogenesis ${ }^{4,9,33,34)}$ (Fig. 2). Clinical trials have been conducted to assess the efficacy of diflunisal on the progression of ATTR. ${ }^{2,3,5)}$ In addition, a new drug, tafamidis, has been developed for the treatment of FAP. Tafamidis binds with high affinity to the T4-binding pockets of the TTR tetramer, leading to stabilization of the tetramer and inhibition of the amyloidogenesis $^{35)}$ (Fig. 2). Tafamidis has been approved in Europe, Japan, Mexico, and Argentina, but only for the treatment of early-stage FAP. ${ }^{2,36)}$ However, tafamidis was unable to halt the progression of the disease in patients with advanced FAP caused by the V30M mutation. ${ }^{37)}$ Therefore, the development of other drugs is of great importance for the treatment of ATTR. TTR stabilizers and/or amyloid fibril disrupters isolated from natural sources may become lead compounds for the treatment of ATTR.

Acknowledgments This work was supported by a Grant to the Amyloidosis Research Committee from the Ministry of Health, Labour, and Welfare, Japan. Support was also received in the form of a JSPS KAKENHI Grant (No. 16K08193) and by the JSPS Core-to-Core Program (B. Asia-Africa Science Platforms). 
Conflict of Interest The authors declare no conflict of interest.

\section{REFERENCES}

1) Johnson SM, Connelly S, Fearns C, Powers ET, Kelly JW. The transthyretin amyloidoses: from delineating the molecular mechanism of aggregation linked to pathology to a regulatory-agencyapproved drug. J. Mol. Biol., 421, 185-203 (2012).

2) Ueda M, Ando Y. Recent advances in transthyretin amyloidosis therapy. Transl. Neurodegener., 3, 19 (2014).

3) Sekijima Y. Transthyretin (ATTR) amyloidosis: clinical spectrum, molecular pathogenesis and disease-modifying treatments. J. Neurol. Neurosurg. Psychiatry, 86, 1036-1043 (2015).

4) Sekijima Y. Recent progress in the understanding and treatment of transthyretin amyloidosis. J. Clin. Pharm. Ther., 39, 225-233 (2014).

5) Ando Y, Coelho T, Berk JL, Cruz MW, Ericzon BG, Ikeda S, Lewis WD, Obici L, Plante-Bordeneuve V, Rapezzi C, Said G, Salvi F. Guideline of transthyretin-related hereditary amyloidosis for clinicians. Orphanet J. Rare Dis., 8, 31 (2013).

6) Benson MD. Leptomeningeal amyloid and variant transthyretins Commentary. Am. J. Pathol., 148, 351-354 (1996).

7) Hammarstrom P, Jiang X, Hurshman AR, Powers ET, Kelly JW. Sequence-dependent denaturation energetics: A major determinant in amyloid disease diversity. Proc. Natl. Acad. Sci. U.S.A., 99 (Suppl. 4), 16427-16432 (2002).

8) Miroy GJ, Lai Z, Lashuel HA, Peterson SA, Strang C, Kelly JW. Inhibiting transthyretin amyloid fibril formation via protein stabilization. Proc. Natl. Acad. Sci. U.S.A., 93, 15051-15056 (1996).

9) Baures PW, Peterson SA, Kelly JW. Discovering transthyretin amyloid fibril inhibitors by limited screening. Bioorg. Med. Chem., 6, 1389-1401 (1998)

10) Hammarström P, Wiseman RL, Powers ET, Kelly JW. Prevention of transthyretin amyloid disease by changing protein misfolding energetics. Science, 299, 713-716 (2003).

11) Costa PP, Figueira AS, Bravo FR. Amyloid fibril protein related to prealbumin in familial amyloidotic polyneuropathy. Proc. Natl. Acad. Sci. U.S.A., 75, 4499-4503 (1978).

12) Ingbar SH. Observations concerning the binding of thyroid hormones by human serum prealbumin. J. Clin. Invest., 42, 143-160 (1963).

13) Auf'mkolk M, Koehrle J, Hesch RD, Ingbar SH, Cody V. Crystal structure of phlorizin and the iodothyronine deiodinase inhibitory activity of phloretin analogues. Biochem. Pharmacol., 35, 22212227 (1986).

14) Smith TJ, Davis FB, Deziel MR, Davis PJ, Ramsden DB, Schoenl M. Retinoic acid inhibition of thyroxine binding to human transthyretin. Biochim. Biophys. Acta, 1199, 76-80 (1994).

15) Zanotti G, D’Acunto MR, Malpeli G, Folli C, Berni R. Crystal structure of the transthyretin-retinoic-acid complex. Eur. J. Biochem., 234, 563-569 (1995).

16) Hammarström P, Schneider F, Kelly JW. Trans-suppression of misfolding in an amyloid disease. Science, 293, 2459-2462 (2001).

17) Maia F, Almeida Mdo R, Gales L, Kijjoa A, Pinto MM, Saraiva MJ, Damas AM. The binding of xanthone derivatives to transthyretin. Biochem. Pharmacol., 70, 1861-1869 (2005).

18) Yokoyama T, Ueda M, Ando Y, Mizuguchi M. Discovery of gamma-mangostin as an amyloidogenesis inhibitor. Sci. Rep., 5, 13570 (2015).

19) Yokoyama T, Kosaka Y, Mizuguchi M. Inhibitory activities of propolis and its promising component, caffeic acid phenethyl ester, against amyloidogenesis of human transthyretin. J. Med. Chem., 57, 8928-8935 (2014).

20) Pullakhandam R, Srinivas PNBS, Nair MK, Reddy GB. Binding and stabilization of transthyretin by curcumin. Arch. Biochem. Biophys., 485, 115-119 (2009).
21) Radovic B, Hussong R, Gerhauser C, Meinl W, Frank N, Becker $\mathrm{H}$, Kohrle J. Xanthohumol, a prenylated chalcone from hops, modulates hepatic expression of genes involved in thyroid hormone distribution and metabolism. Mol. Nutr. Food Res., 54 (Suppl. 2), S225-S235 (2010).

22) Green NS, Foss TR, Kelly JW. Genistein, a natural product from soy, is a potent inhibitor of transthyretin amyloidosis. Proc. Natl. Acad. Sci. U.S.A., 102, 14545-14550 (2005).

23) Trivella DB, dos Reis CV, Lima LM, Foguel D, Polikarpov I. Flavonoid interactions with human transthyretin: combined structural and thermodynamic analysis. J. Struct. Biol., 180, 143-153 (2012).

24) Yokoyama T, Kosaka Y, Mizuguchi M. Crystal structures of human transthyretin complexed with glabridin. J. Med. Chem., 57, 10901096 (2014).

25) Ferreira N, Cardoso I, Domingues MR, Vitorino R, Bastos M, Bai GY, Saraiva MJ, Almeida MR. Binding of epigallocatechin-3-gallate to transthyretin modulates its amyloidogenicity. FEBS Lett., 583, 3569-3576 (2009).

26) Miyata M, Sato T, Kugimiya M, Sho M, Nakamura T, Ikemizu S, Chirifu M, Mizuguchi M, Nabeshima Y, Suwa Y, Morioka H, Arimori T, Suico MA, Shuto T, Sako Y, Momohara M, Koga T, Morino-Koga S, Yamagata Y, Kai H. The crystal structure of the green tea polyphenol (-)-epigallocatechin gallate-transthyretin complex reveals a novel binding site distinct from the thyroxine binding site. Biochemistry, 49, 6104-6114 (2010).

27) Cardoso I, Saraiva MJ. Doxycycline disrupts transthyretin amyloid: evidence from studies in a FAP transgenic mice model. FASEB J., 20, 234-239 (2006).

28) Gupta S, Babu P, Surolia A. Biphenyl ethers conjugated CdSe/ZnS core/shell quantum dots and interpretation of the mechanism of amyloid fibril disruption. Biomaterials, 31, 6809-6822 (2010).

29) Gupta S, Chhibber M, Sinha S, Surolia A. Design of mechanismbased inhibitors of transthyretin amyloidosis: Studies with biphenyl ethers and new structural templates. J. Med. Chem., 50, 5589-5599 (2007).

30) Peterson SA, Klabunde T, Lashuel HA, Purkey H, Sacchettini JC, Kelly JW. Inhibiting transthyretin conformational changes that lead to amyloid fibril formation. Proc. Natl. Acad. Sci. U.S.A., 95, 12956-12960 (1998).

31) Baures PW, Oza VB, Peterson SA, Kelly JW. Synthesis and evaluation of inhibitors of transthyretin amyloid formation based on the non-steroidal anti-inflammatory drug, flufenamic acid. Bioorg. Med. Chem., 7, 1339-1347 (1999).

32) Oza VB, Smith C, Raman P, Koepf EK, Lashuel HA, Petrassi HM, Chiang KP, Powers ET, Sachettinni J, Kelly JW. Synthesis, structure, and activity of diclofenac analogues as transthyretin amyloid fibril formation inhibitors. J. Med. Chem., 45, 321-332 (2002).

33) Adamski-Werner SL, Palaninathan SK, Sacchettini JC, Kelly JW. Diflunisal analogues stabilize the native state of transthyretin. Potent inhibition of amyloidogenesis. J. Med. Chem., 47, 355-374 (2004).

34) Klabunde T, Petrassi HM, Oza VB, Raman P, Kelly JW, Sacchettini JC. Rational design of potent human transthyretin amyloid disease inhibitors. Nat. Struct. Biol., 7, 312-321 (2000).

35) Bulawa CE, Connelly S, Devit M, Wang L, Weigel C, Fleming JA, Packman J, Powers ET, Wiseman RL, Foss TR, Wilson IA, Kelly JW, Labaudiniere R. Tafamidis, a potent and selective transthyretin kinetic stabilizer that inhibits the amyloid cascade. Proc. Natl. Acad. Sci. U.S.A., 109, 9629-9634 (2012).

36) Cruz MW, Benson MD. A Review of Tafamidis for the Treatment of Transthyretin-Related Amyloidosis. Neurol. Ther., 4, 61-79 (2015)

37) Lozeron $P$, Theaudin M, Mincheva Z, Ducot B, Lacroix C, Adams D, French Network for FAP (CORNAMYL). Effect on the disability and safety of Tafamidis in late onset Met30 transthyretin familial amyloid polyneuropathy. Eur. J. Neurol., 20, 1539-1545 (2013). 